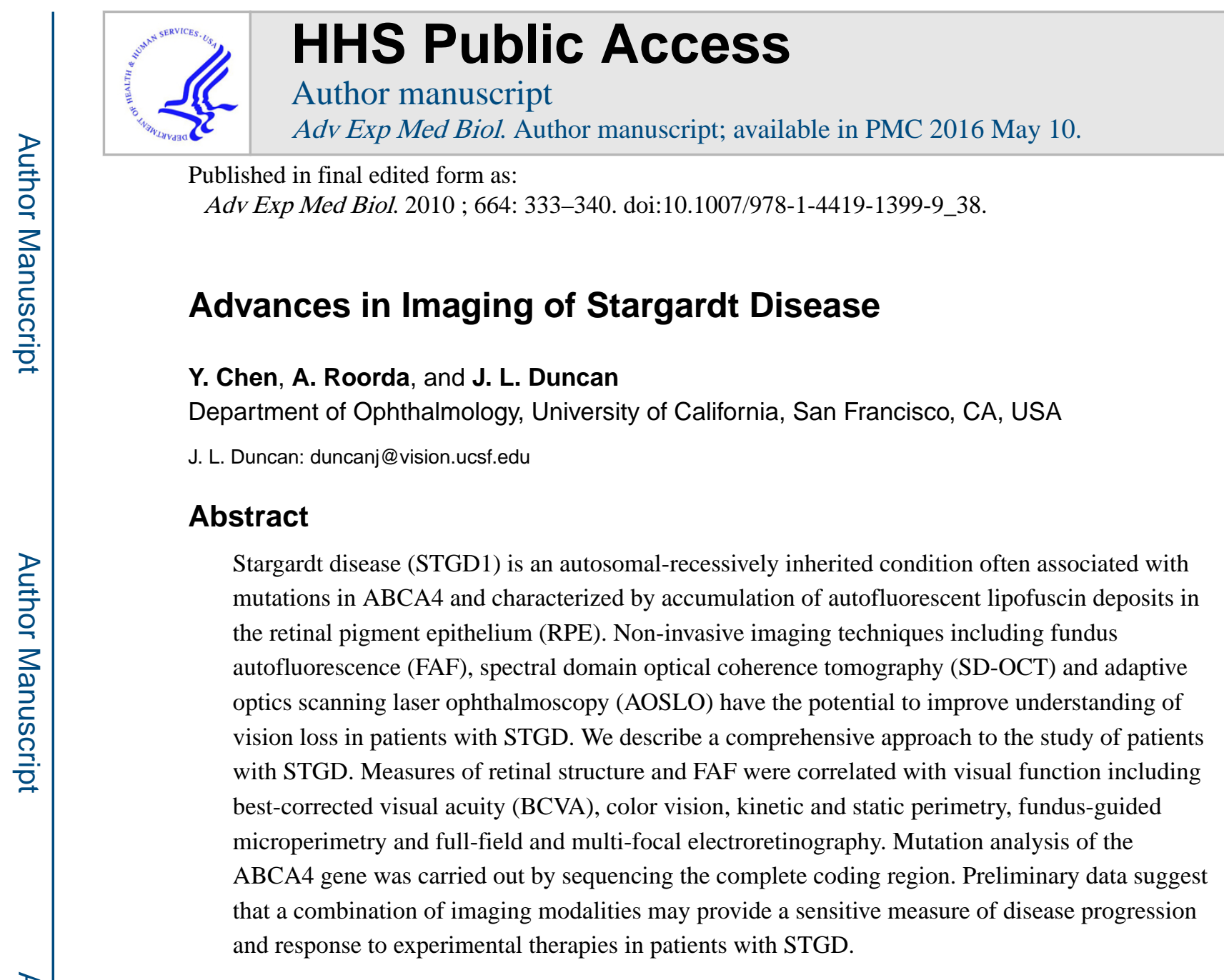

\title{
38.1 Introduction
}

Stargardt disease/fundus flavimaculatus (STGD/FF) is the most common form of hereditary macular dystrophy in childhood, affecting 1 in 10,000 individuals (Bither and Berns 1988). The progression of vision loss in autosomal recessive STGD is usually rapid in childhood and young adulthood, but may be unpredictable and often does not correlate with the severity of fundus lesions (Klevering et al. 2002; Klevering et al. 2002; Rotenstreich et al. 2003). Fundus photographs of early disease range from a beaten-bronze appearance to atrophy, often presenting with characteristic yellow-white flecks at the level of the retinal pigment epithelium (RPE) (Armstrong et al. 1998). Histology shows the accumulation of lipofuscin, a lipid-containing fluorophoric by-product of photoreceptor digestion, inside the RPE (Sparrow and Boulton 2005). In advanced STGD, lipofuscin is associated with atrophy of photoreceptors, RPE, and choroidal vasculature concurrent with central vision loss (Rotenstreich et al. 2003). Fluorescein angiograms of STGD/FF often show a characteristic 'dark choroid,' superimposed by non-homogenous hyperfluorescent regions in the posterior pole (Fishman et al. 1987).

Eighty percent of patients with clinical STGD/FF have mutations in the ABCA4 gene, a phospholipid flippase found in the outer disc segments of photoreceptors involved in retinoid 
recycling (Allikmets 1997; Kitiratschky et al. 2008). ABCA4 defects lead to the intracellular accumulation of A2E in RPE cells (Radu et al. 2004). A2E is cytotoxic to the RPE in high concentrations (Sparrow et al. 2003) and can be visualized in living eyes using fundus autofluorescence (FAF).

This manuscript will review three non-invasive imaging techniques used in diagnosing and characterizing STGD/FF, fundus autofluorescence (FAF), optical coherence tomography (OCT), and adaptive-optics scanning laser ophthalmoscopy (AOSLO), and describe methods to evaluate cone and RPE cell structure in patients with STGD/FF.

\subsection{Fundus Autofluorescence}

FAF, a non-invasive imaging technique used to visualize lipofuscin, originates from the RPE layer; its distribution pattern in the normal retina is elevated in the parafoveal area, reduced in the fovea and towards the periphery, and absent over the optic disc and large blood vessels (Delori et al. 1995a; Delori et al. 2001; von Ruckmann et al. 1995; von Ruckmann et al. 1997). Abnormally increased FAF suggests RPE dysfunction, while decreased FAF indicates RPE atrophy and pho-toreceptor death (Lois et al. 2000; von Ruckmann et al. 1995). Most FAF images are acquired with a confocal scanning laser ophthalmoscopy system, which gathers light from a single optical plane, effectively reducing AF from sources anterior to the retina (Sharp et al. 2004).

Abnormalities in AF intensity, texture, and/or topographic distribution can be found in a spectrum of macular degenerative diseases (Cideciyan et al. 2004; Delori et al. 1995; Delori et al. 2000; Lois et al. 2004; von Ruckmann et al. 1997; Wabbels et al. 2006). The abnormal increase of RPE lipofuscin is likely the first detectable change in STGD/FF, with its continuous accumulation used as a likely marker for early disease progression (Cideciyan et al. 2004). Studies of early STGD/FF have consistently demonstrated abnormal AF, although with wide variation in pattern and degree. In some patients, AF images reveal areas of atrophy or flecks not seen on fundus photographs, suggesting its potential in detecting early disease (Boon et al. 2008; Lois et al. 1999). Recent studies have correlated FAF to visual function. Lois et al. (2004) demonstrated that STGD eyes without photoreceptor dysfunction as measured by pattern ERG had either normal AF throughout the macula or normal AF at the fovea surrounded by focally increased AF. In contrast, those with photoreceptor dysfunction had variably decreased $\mathrm{AF}$ at the fovea, presumably reflecting advanced disease with RPE and photoreceptor atrophy. Cideciyan et al. (2004) proposed a disease sequence model for ABCA4-associated retinopathies based on FAF findings. Early detectable disease is characterized by diffusely increased FAF in the posterior pole, and is followed by the appearance of focally increased FAF flecks in the perifoveal region. In late disease, FAF decreases due to dysfunction and eventual loss of RPE cells, leading to photoreceptor degeneration and death (Cideciyan et al. 2004).

Most FAF studies have relied on qualitative descriptions, characterizing the intensity as decreased, normal, or increased, with significant inter-observer variability (Lois et al. 2000; Sunness et al. 2006). Images captured by different systems show variability in absolute signal intensity and contrast, due to discrepancies in detector gain, argon laser amplification 
value, and normalization value (Bellmann et al. 2003). Furthermore, FAF intensity cannot be directly correlated with lipofuscin accumulation, due to absorption of fluorescence by a number of ocular structures, including melanin pigments, nerve fibers, capillaries, and lens opacities (Delori et al. 2001).

\subsection{OCT}

OCT is a non-invasive technique that provides high-resolution axial images of the retina, with depth resolution better than $3 \mu \mathrm{m}$ (Drexler and Fujimoto 2008; Podoleanu and Rosen 2008). A low coherence infrared source is used to image the retina and a portion of coherent backscattered light is detected using an optical interferometer (Podoleanu and Rosen 2008). Depth and intensity information from captured signal light are converted digitally to visualize structural morphology of intraretinal layers (Drexler and Fujimoto 2008). Spectral domain OCT (SD-OCT) is the most advanced OCT method available and provides high resolution, fast scanning speeds, high repeatability, and the capacity for transverse C-scans and the 3D mapping of single retinal layers (Forte et al. 2008; Gupta et al. 2008; Leung et al. 2008).

Macular thickness and other retinal architectural OCT features provide useful information on the transverse and axial location of retinal lesions, atrophy, and other microstructural changes seen in STGD. OCT has revealed disruption or absence of inner and outer photoreceptor segment layers with or without thinning of other intraretinal layers, and visual acuity loss correlated with central foveal thickness in atrophic areas in patients with STGD (Ergun et al. 2005).

\subsection{Adaptive Optics Scanning Laser Ophthalmoscope}

AOSLO uses a wavefront sensor to measure ocular aberrations and compensates for them using a deformable mirror, providing non-invasive, high-resolution images of the retina (Roorda et al. 2002; Zhang et al. 2006). Achieving higher contrast and a transverse resolution of $2 \mu \mathrm{m}$, it is capable of imaging individual cones and single leukocytes moving through retinal capillaries (Martin and Roorda 2005; Roorda et al. 2002). Multiple frames from the same video can be added together and averaged to improve the signal-to-noise ratio, producing clearer images where most, if not all, photoreceptors are resolved (Zhang et al. 2006). Cones in healthy eyes are clearly visualized as bright spots arranged in organized hexagonal arrays with regular spacing, while cones in eyes with retinopathies show abnormal morphology, spacing, and packing patterns (Choi et al. 2006; Duncan et al. 2007; Roorda et al. 2007; Wolfing et al. 2006; Yoon et al. 2008).

Images of patients with inherited retinal degenerations, using both flood-illuminated $\mathrm{AO}$ ophthalmoscopes (Choi et al. 2006) and AOSLO systems (Duncan et al. 2007; Wolfing et al. 2006), have observed increased cone spacing/reduced cone density compared to normals, as well as hyper-reflective lesions in regions where cones and RPE were absent clinically. AO images of a patient with juvenile macular dystrophy showed an irregular cone mosaic where retinal sensitivity was reduced (Choi et al. 2006). A regular array of RPE cells in an annular region surrounding the cone-preserved fovea was visualized in 2 eyes with cone-rod 
dystrophy (Roorda et al. 2007). AOSLO imaging of members of a family with a mitochondrial mutation causing NARP syndrome (neurogenic muscle weakness, ataxia, and retinitis pigmentosa) showed three predominant cone spacing patterns: normal, increased within a contiguous mosaic, and patchy cone loss with increased spacing, the latter of which correlated with the most severe impairment in visual function (Yoon et al. 2008).

The use of AOSLO to study patients with inherited retinal degenerations both during disease progression and in response to experimental therapies requires accurate and reliable identification of not only normal cones but of also irregularly shaped and packed cones. Although software-based automated algorithms have achieved over $90 \%$ agreement between automated and manual methods ( $\mathrm{Li}$ and Roorda 2007), unambiguous identification of cones is not always reliable using either method. Lack of visible cones may not always indicate photoreceptor loss, but could instead be due to poor image resolution or scattering from media anterior to the photoreceptors. Conversely, small, bright features in an AOSLO image are not always photoreceptors. For this reason, cone spacing is reliably quantified only in regions where cone mosaics can be identified unambiguously. The criteria for an unambiguous cone array is the appearance of multiple, similar-sized features arranged in a close-packed array, in addition to the presence of the expected laminar appearance of the photoreceptor inner and outer segment layers in a corresponding SD-OCT cross-section. An example of four imaging modalities used to image the nasal aspect of a normal fovea is illustrated in Fig. 38.1.

Although each of the reviewed imaging modalities has been established as a reliable diagnostic tool, a combination of imaging modalities including evaluation of structure on a cellular level may yield the most comprehensive characterization of the disease phenotype in STGD/FF patients. High-resolution images of the macula were obtained using AOSLO and SD-OCT in 5 patients with STGD and 10 age-similar normal subjects. A STGD patient with vision reduced to 20/200 and eccentric fixation nasal to the anatomic fovea shows regions of irregular and reduced FAF that correlate with increased cone spacing and loss of the photoreceptor inner and outer segment layers closer to the anatomic fovea on SD-OCT, while cone spacing is preserved adjacent to the optic nerve where FAF is more uniform (Fig. 38.2).

\subsection{Conclusion}

$\mathrm{STGD/FF}$ is a complex retinal degenerative disease with variable disease presentation and progression. Better understanding of disease pathogenesis will require longitudinal data integration and analyses using multiple parameters, many of which can be acquired through the three imaging modalities reviewed above. Each technology has value in identifying disease severity and progression, but each also has intrinsic limitations that the other methods can complement. FAF can track lipofus-cin accumulation, OCT can visualize crosssectional retinal structure and AOSLO can directly visualize photoreceptor morphology to yield valuable insights into the temporal and topographic patterns of cone death. Understanding disease progression on a high-resolution scale will be essential in developing and monitoring response to therapies as they become available. 


\section{Acknowledgments}

Supported by a Career Development Award, Physician Scientist Award and Unrestricted Grant from Research to Prevent Blindness (JLD); a Career Development Award and Clinical Center Grant from the Foundation Fighting Blindness (JLD, AR); NIH-NEI grants EY00415, EY002162 (JLD), EY014375 (AR); That Man May See, Inc. (JLD); The Bernard A. Newcomb Macular Degeneration Fund (JLD); Hope for Vision (JLD); and the Karl Kirchgessner Foundation (JLD).

\section{References}

Allikmets R. A photoreceptor cell-specific ATP-binding transporter gene (ABCR) is mutated in recessive Stargardt macular dystrophy. Nat Genet. 1997; 17:122. [PubMed: 9288113]

Armstrong JD, Meyer D, Xu S, et al. Long-term follow-up of Stargardt's disease and fundus flavimaculatus. Ophthalmology. 1998; 105:448-457. [PubMed: 9499775]

Bellmann C, Rubin GS, Kabanarou SA, et al. Fundus autofluorescence imaging compared with different confocal scanning laser ophthalmoscopes. Br J Ophthalmol. 2003; 87:1381-1386. [PubMed: 14609839]

Bither PP, Berns LA. Stargardt's disease: a review of the literature. J Am Optom Assoc. 1988; 59:106111. [PubMed: 3283201]

Boon CJ, Jeroen Klevering B, Keunen JE, et al. Fundus autofluorescence imaging of retinal dystrophies. Vision Res. 2008; 48:2569-2577. [PubMed: 18289629]

Choi SS, Doble N, Hardy JL, et al. In vivo imaging of the photoreceptor mosaic in retinal dystrophies and correlations with visual function. Invest Ophthal Vis Sci. 2006; 47:2080-2092. [PubMed: 16639019]

Cideciyan AV, Aleman TS, Swider M, et al. Mutations in ABCA4 result in accumulation of lipofuscin before slowing of the retinoid cycle: a reappraisal of the human disease sequence. Hum Mol Genet. 2004; 13:525-534. [PubMed: 14709597]

Delori FC, Dorey CK, Staurenghi G, et al. In vivo fluorescence of the ocular fundus exhibits retinal pigment epithelium lipofuscin characteristics. Invest Ophthal Vis Sci. 1995a; 36:718-729. [PubMed: 7890502]

Delori FC, Fleckner MR, Goger DG, et al. Autofluorescence distribution associated with drusen in age-related macular degeneration. Invest Ophthal Vis Sci. 2000; 41:496-504. [PubMed: 10670481]

Delori FC, Goger DG, Dorey CK. Age-related accumulation and spatial distribution of lipofuscin in RPE of normal subjects. Invest Ophthal Vis Sci. 2001; 42:1855-1866. [PubMed: 11431454]

Delori FC, Staurenghi G, Arend O, et al. In vivo measurement of lipofuscin in Stargardt's diseasefundus flavimaculatus. Invest Ophthal Vis Sci. 1995b; 36:2327-2331. [PubMed: 7558729]

Drexler W, Fujimoto JG. State-of-the-art retinal optical coherence tomography. Prog Retin Eye Res. 2008; 27:45-88. [PubMed: 18036865]

Duncan JL, Zhang Y, Gandhi J, et al. High-resolution imaging with adaptive optics in patients with inherited retinal degeneration. Invest Ophthal Vis Sci. 2007; 48:3283-3291. [PubMed: 17591900]

Ergun E, Hermann B, Wirtitsch M, et al. Assessment of central visual function in Stargardt's disease/ fundus flavimaculatus with ultrahigh-resolution optical coherence tomography. Invest Ophthal Vis Sci. 2005; 46:310-316. [PubMed: 15623790]

Fishman GA, Farber M, Patel BS, et al. Visual acuity loss in patients with Stargardt's macular dystrophy. Ophthalmology. 1987; 94:809-814. [PubMed: 3658351]

Forte R, Cennamo GL, Finelli ML, et al. Comparison of time domain stratus OCT and spectral domain SLO/OCT for assessment of macular thickness and volume. Eye (London). 2009; 23:2071-2078.

Gupta V, Gupta P, Singh R, et al. Spectral-domain cirrus high-definition optical coherence tomography is better than time-domain stratus optical coherence tomography for evaluation of macular pathologic features in uveitis. Am J Ophthalmol. 2008; 145:1018-1022. [PubMed: 18343349]

Kitiratschky VB, Grau T, Bernd A, et al. ABCA4 gene analysis in patients with autosomal recessive cone and cone rod dystrophies. Eur J Hum Genet. 2008; 16:812-819. [PubMed: 18285826] 
Klevering BJ, Blankenagel A, Maugeri A, et al. Phenotypic spectrum of autosomal recessive cone-rod dystrophies caused by mutations in the ABCA4 (ABCR) gene. Invest Ophthal Vis Sci. 2002; 43:1980-1985. [PubMed: 12037008]

Leung CK, Cheung CY, Weinreb RN, et al. Comparison of macular thickness measurements between time domain and spectral domain optical coherence tomography. Invest Ophthal Vis Sci. 2008; 49:4893-4897. [PubMed: 18450592]

Li KY, Roorda A. Automated identification of cone photoreceptors in adaptive optics retinal images. J Opt Soc Am A Opt Image Sci Vis. 2007; 24:1358-1363. [PubMed: 17429481]

Lois N, Halfyard AS, Bird AC, et al. Quantitative evaluation of fundus autofluorescence imaged "in vivo" in eyes with retinal disease. Br J Ophthalmol. 2000; 84:741-745. [PubMed: 10873986]

Lois N, Halfyard AS, Bird AC, et al. Fundus autofluorescence in Stargardt macular dystrophy-fundus flavimaculatus. Am J Ophthalmol. 2004; 138:55-63. [PubMed: 15234282]

Lois N, Holder GE, Fitzke FW, et al. Intrafamilial variation of phenotype in stargardt macular dystrophy-fundus flavimaculatus. Invest Ophthal Vis Sci. 1999; 40:2668-2675. [PubMed: 10509664]

Martin JA, Roorda A. Direct and noninvasive assessment of parafoveal capillary leukocyte velocity. Ophthalmology. 2005; 112:2219-2224. [PubMed: 16257054]

Podoleanu AG, Rosen RB. Combinations of techniques in imaging the retina with high resolution. Prog Retin Eye Res. 2008; 27:464-499. [PubMed: 18495519]

Radu RA, Mata NL, Bagla A, et al. Light exposure stimulates formation of A2E oxiranes in a mouse model of Stargardt's macular degeneration. Proc Natl Acad Sci USA. 2004; 101:5928-5933. [PubMed: 15067110]

Roorda A, Romero-Borja F, Donnelly W III, et al. Adaptive optics scanning laser ophthalmoscopy. Opt Express. 2002; 10:405-412. [PubMed: 19436374]

Roorda A, Zhang Y, Duncan JL. High-resolution in vivo imaging of the RPE mosaic in eyes with retinal disease. Invest Ophthal Vis Sci. 2007; 48:2297-2303. [PubMed: 17460294]

Rotenstreich Y, Fishman GA, Anderson RJ. Visual acuity loss and clinical observations in a large series of patients with stargardt disease. Ophthalmology. 2003; 110:1151-1158. [PubMed: 12799240]

Sharp PF, Manivannan A, Xu H, et al. The scanning laser ophthalmoscope-a review of its role in bioscience and medicine. Phys Med Biol. 2004; 49:1085-1096. [PubMed: 15128191]

Sparrow JR, Boulton M. RPE lipofuscin and its role in retinal pathobiology. Exp Eye Res. 2005; 80:595-606. [PubMed: 15862166]

Sparrow JR, Fishkin N, Zhou J, et al. A2E, a byproduct of the visual cycle. Vision Res. 2003; 43:2983-2990. [PubMed: 14611934]

Sunness JS, Ziegler MD, Applegate CA. Issues in quantifying atrophic macular disease using retinal autofluorescence. Retina. 2006; 26:666-672. [PubMed: 16829810]

von Ruckmann A, Fitzke FW, Bird AC. Distribution of fundus autofluorescence with a scanning laser ophthalmoscope. Br J Ophthalmol. 1995; 79:407-412. [PubMed: 7612549]

von Ruckmann A, Fitzke FW, Bird AC. Fundus autofluorescence in age-related macular disease imaged with a laser scanning ophthalmoscope. Invest Ophthal Vis Sci. 1997; 38:478-486. [PubMed: 9040481]

Wabbels B, Demmler A, Paunescu K, et al. Fundus autofluorescence in children and teenagers with hereditary retinal diseases. Graefes Arch Clin Exp Ophthalmol. 2006; 244:36-45. [PubMed: 16034607]

Wolfing JI, Chung M, Carroll J, et al. High-resolution retinal imaging of cone-rod dystrophy. Ophthalmology. 2006; 113:1019, e1. [PubMed: 16650474]

Yoon MK, Roorda A, Zhang Y, et al. Adaptive optics scanning laser ophthalmoscopy images demonstrate abnormal cone structure in a family with the mitochondrial DNA T8993C mutation. Invest Ophthal Vis Sci. 2009; 50:1838-1847. [PubMed: 18997096]

Zhang Y, Poonja S, Roorda A. MEMS-based adaptive optics scanning laser ophthalmoscopy. Opt Lett. 2006; 31:1268-1270. [PubMed: 16642081] 

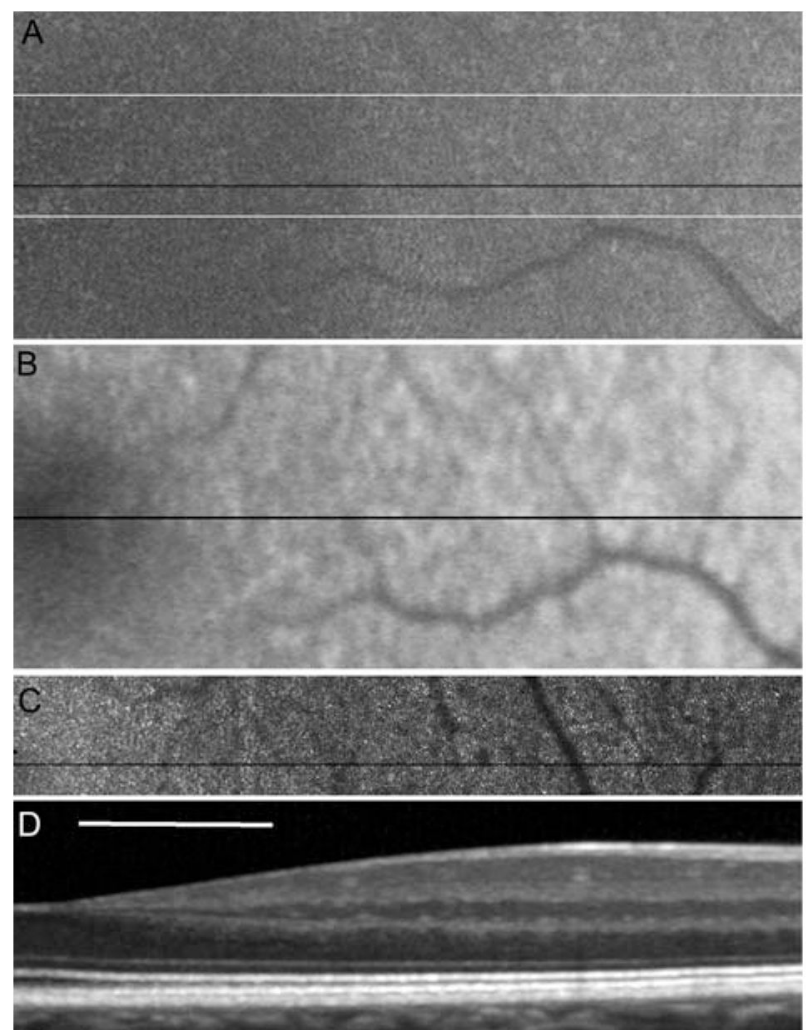

Fig. 38.1.

Images of the right eye of a normal subject. The fovea is on the left edge of the image. Scale bar is $1^{\circ}(\sim 300 \mu)$ (a) fundus photograph, (b) FAF, (c) AOSLO and (d) SD-OCT. The black lines on $\mathbf{a}, \mathbf{b}$ and $\mathbf{c}$ indicate the exact location of the SD-OCT scan. The thin white lines on (a) indicate the exact bounds for the AOSLO image overlay. Image (b) shows the expected decrease in AF toward the fovea. The AOSLO image shows resolved photoreceptors with increasing spacing with distance from the fovea (left to right). The OCT image shows the typical layered structure, including well-defined inner and outer segments expected for a healthy photoreceptor layer 


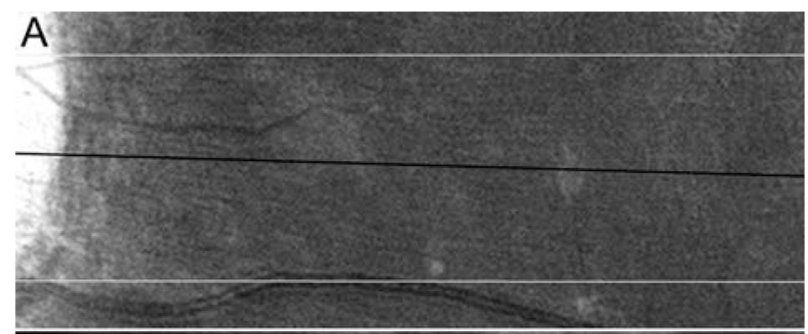

\section{B}

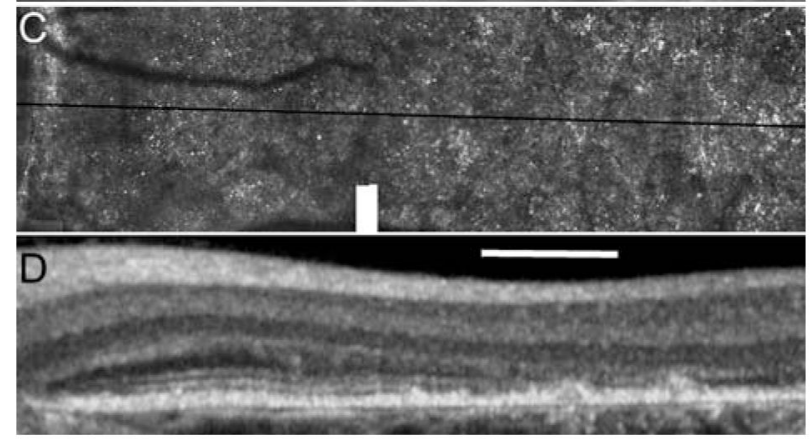

Fig. 38.2.

Images of the left eye of a STGD patient. The edge of the optic disc is on the left edge of the image. The scale bar is $1^{\circ}(\sim 300 \mu)$ (a) fundus photograph (b) FAF, (c) AOSLO and (d) SDOCT. The dark lines indicate the location of the OCT cross section. The thin white lines on (a) indicate the bounds of the AOSLO frame. FAF is mottled throughout the image with the exception a band around the optic disc. Uniform FAF corresponds with a regular array of cone photoreceptors in the AOSLO image and is further confirmed in the SD-OCT image by the presence of a laminar structure corresponding to photoreceptor inner and outer segments 